\title{
¿QUÉ ES LA PROPIEDAD?
}

\author{
What is Property?
}

\author{
ADRIANO ZAMBON*
}

\section{Resumen:}

Este artículo tiene como objetivo proponer una respuesta a la pregunta: ¿Qué es la propiedad? Inicialmente, se delinearán dos usos diferentes del término "propiedad" y se intentará mostrar cómo pueden ser explicados de dos maneras diferentes. Las dos explicaciones comparten algunos aspectos, cuyo análisis conducirá al sentido mínimo de la palabra "propiedad". Este sentido, que puede verse como el concepto de propiedad y como un tipo específico de respuesta a la pregunta inicial, se distinguirá de las concepciones de propiedad. Luego, se examinará la relación entre este concepto y las nociones de persona y bien, junto con un problema relacionado con la transferencia de la propiedad. Finalmente, se formularán algunas conclusiones generales sobre la utilidad del tipo de respuesta aquí propuesto a la pregunta inicial.

Palabras clave: concepto; concepción; normas o reglas; posiciones jurídicas subjetivas; propiedad

\begin{abstract}
:
This paper aims to provide an answer to the question: What is property? Initially, two different uses of the term "property" will be outlined, and an attempt will be made to show how they can be explained in two different ways. The two explanations share some aspects, the analysis of which will lead to the minimal sense of the word "property". This sense, which can be seen as the concept of property and as a specific type of answer to the initial question, will be distinguished from the conceptions of property. Then, the relationship between this concept and the notions of person and good will be analyzed, together with a problem related to the transfer of property. Finally, some general conclusions regarding the usefulness of the type of answer here provided to the initial question will be formulated.
\end{abstract}

Keywords: Concept; Conception; Norms Or Rules; Property; Subjective Legal Positions

* Universidad de Milán, Italia (adriano.zambon@unimi.it). Artículo recibido el 15 de marzo de 2020 y aceptado para su publicación el 2 de junio de 2020. 


\section{TRES PROBLEMAS}

Cuando se trata de propiedad, es bueno distinguir primero tres problemas diferentes que le conciernen. ${ }^{1}$

El primer problema, el cual trataré en este artículo, es el problema de qué es la propiedad. El segundo problema es el problema de la justificación de la propiedad y se refiere a las razones para sostener que la existencia de la propiedad es justa. ${ }^{2}$ El tercer y último problema es el problema de la distribución de la propiedad: ¿cuál es la forma correcta de distribuir la propiedad a las personas?

Como se mencionó, me ocuparé del primer problema. La razón de esta elección es que este problema debe resolverse necesariamente antes de que se pueda tratar de resolver los otros dos. De hecho, ellos requieren que las ideas sobre lo que es justo e injusto se apliquen a la propiedad, pero no es posible aplicar tales ideas a la propiedad si no se ha establecido qué es la propiedad. Es por esta razón que el primer problema que debe enfrentar la filosofía del derecho (cuando se ocupa de la propiedad) solo puede ser este.

Para este fin, procederé de la siguiente manera. Primero, analizaré algunos modos de usar el término "propiedad" e intentaré comprender cuál es el propósito de cada uno de estos usos. En segundo lugar, mostraré cómo se pueden avanzar diferentes tesis sobre qué es la propiedad, las cuales se pueden utilizar para explicar, de diferentes maneras, los usos del término "propiedad" considerados anteriormente. En tercer lugar, intentaré resaltar que todas las tesis en cuestión representan diferentes descripciones de la propiedad y que se pueden describir utilizando una sola fórmula. Esta posibilidad significa que esta fórmula es una descripción de la propiedad compartida por todas las tesis en cuestión. Completar estas operaciones dará lugar a una respuesta a la pregunta: “QQué es la propiedad?”.

Tal respuesta, sin embargo, no se encuentra exenta de problemas: nos permite encontrar lo que puede entenderse como el concepto de propiedad, que tendrá que distinguirse de las concepciones de propiedad. Después de descri-

1 Sobre esta distinción, véase HART (1968), p. 4.

2 Esta es solo una descripción genérica del problema de la justificación de la propiedad, porque parece posible distinguir tres tipos diferentes de justificación de la propiedad: una justificación general (que responde a la pregunta de por qué debería haber alguna propiedad en absoluto), una justificación específica (que responde a la pregunta de por qué debería haber un tipo específico de propiedad) y una justificación particular (que responde a la pregunta de por qué un individuo en particular debería tener propiedad). Esta distinción proviene de BECKER (1977), p. 23, que, sin embargo, la aplica a la justificación de los derechos de propiedad. 
bir esta distinción, será posible ver cómo ciertos elementos relacionados con el concepto de propiedad son externos a este concepto y también por qué este concepto puede ser útil.

\section{DOS USOS DEL TÉRMINO "PROPIEDAD”}

Una buena manera de comenzar a preguntarse sobre qué es la propiedad es preguntarse cómo usamos el término "propiedad". Si observamos cómo se usa esta palabra en el lenguaje ordinario y en el lenguaje jurídico, podemos ver que sus usos son principalmente dos. ${ }^{3}$

El primer uso se encuentra en frases como "Este contrato se refiere a la propiedad de esa tierra" o "Esta ley se refiere a la propiedad de algoritmos". En enunciados de este tipo, el término "propiedad" parece designar algo que tiene como objeto un bien material (como la tierra) o un bien intangible (como un algoritmo). La propiedad, en tales casos, puede verse como la propiedad de un bien. En consecuencia, es algo que concierne a un bien.

En segundo lugar, la palabra "propiedad" se puede usar para designar un bien material o inmaterial, por ejemplo, en enunciados como "Esta tierra es propiedad privada", "Este bolígrafo es mi propiedad" y "Esta información es propiedad de la empresa". En tales casos, el término no es usado para indicar algo que concierne a un bien, sino al bien mismo.

Por lo tanto, las dos cosas diferentes que el término "propiedad" puede designar son:

1. algo que tiene como objeto un bien material o un bien inmaterial;

2. un bien material o un bien inmaterial.

¿Hay alguna conexión entre estos dos usos? Para entender si la respuesta a esta pregunta es afirmativa, es posible considerar la primera cosa que el término puede designar, ya que por ahora es excesivamente genérica (a diferencia de la

3 Sin embargo, es importante especificar que no me referiré al uso del término "propiedad" que tiene como objetivo designar la característica o las características de algo (es un uso que se puede encontrar en frases como "Este compuesto tiene propiedades milagrosas"). Sobre este punto, véase SNARE (1972), p. 200. 
segunda). ¿Qué es ese algo que el término "propiedad" designa en el primero de los dos usos considerados y que concierne a un bien?

\title{
III. LA INMATERIALIDAD DE LA PROPIEDAD
}

Una primera pregunta que debe abordarse para llegar a una respuesta a la pregunta que se acaba de hacer es si lo que "propiedad" designa en los casos mencionados anteriormente es algo material o inmaterial. La opción correcta parece ser la segunda y no es raro encontrar afirmaciones de su obviedad. A pesar de esto, podemos preguntarnos qué argumentos se pueden usar para defenderla. Tal argumento se puede encontrar en el "Blue Bentham": 4

\begin{abstract}
No hay imagen, no hay pintura, no hay rasgo visible que pueda expresar esta relación que constituye la propiedad: esto nace de que es no material, sino metafísica, y una pura concepción del entendimiento.

Tener la cosa entre sus manos, guardarla, fabricarla, venderla, transformarla, emplearla; todas estas circunstancias físicas no dan aun una idea clara de la propiedad; porque una pieza de tela que está en las indias puede ser mía mientras el vestido que llevo puede no serlo, y el alimento que se ha incorporado en mi sustancia puede ser de otro a quien debo dar cuenta de él. ${ }^{5}$
\end{abstract}

Bentham parece asumir que hay una equivalencia entre lo material y lo visible. Afirma luego que la existencia de la propiedad sobre un bien no constituye algo que pueda percibirse por la vista (y el hecho de que Bentham hable de propiedad para indicar algo distinto de un bien indica que el uso del término al que se refiere no es el uso a través del cual se designa un bien material o un bien inmaterial). Esto se demuestra por el hecho de que esas circunstancias físicas que normalmente están asociadas a la propiedad y pueden percibirse a la vista no nos dicen nada sobre la propiedad de ese bien: uno puede verlas, pero no puede ver nada de la propiedad de un bien. En consecuencia, en virtud de la equivalencia asumida inicialmente entre lo visible y lo material, la propiedad no puede ser algo material.

La tesis de la invisibilidad de la propiedad es apoyada, de manera análoga, por Félix S. Cohen en el siguiente intercambio de palabras entre B y C:

4 JenKs (1932), p. 289

5 Bentham (1821), p. 263 [el texto sigue reglas ortográficas antiguas]. 
B. Well, here is a book that is my property. You can see it, feel it, weigh it. What better proof could there be of the existence of private property?

C. I can see the shape and color of the book very well, but I don't see its propertiness. What sort of evidence can you put forward to show that the book is your property? ${ }^{6}$

Un análogo argumento se puede encontrar en Frank Snare, cuando escribe que "a stolen apple doesn't look any different from any other apple", 7 que significa que la existencia de la propiedad sobre un bien visible no es visible.

\section{LA PROPIEDAD COMO UNA O MÁS POSICIONES JURÍDICAS SUBJETIVAS}

Después de aceptar que la propiedad es algo inmaterial, podríamos decir que es un derecho subjetivo. Sobre la base de esta respuesta, el término "propiedad", en su primer uso, designa un derecho subjetivo sobre un bien. Esta respuesta permite explicar el segundo uso del término de esta manera: el término "propiedad" designaría el bien sobre el cual existe el derecho en cuestión. ${ }^{8}$

Sin embargo, esta respuesta genera otra pregunta: ¿cuáles son las características del derecho identificable con la propiedad? En otras palabras, ¿cuáles son los contenidos de este derecho? Es decir, más simplemente, ¿qué derecho es? Puede haber respuestas diferentes a esta pregunta. Se podría afirmar, por ejemplo, que la propiedad es el derecho al uso exclusivo de un bien ${ }^{9}$ y luego especificar qué significa la expresión "uso exclusivo de un bien", al decir, por ejemplo, que el derecho al uso exclusivo de un bien implica la posibilidad de consumir o destruir el bien y la exclusión de otros del acceso al bien. Desde cierto punto de vista, se puede decir que las especificaciones de este tipo no conducen a un cambio de la tesis según la cual la propiedad es un único derecho (esto es, el derecho al uso exclusivo de un bien).

Sin embargo, desde otro punto de vista, tales especificaciones pueden conducir a un cambio parcial de la respuesta proporcionada inicialmente. En otras palabras,

6 Cohen (1954), p. 359.

7 Snare (1972), p. 200.

8 Una expresión de esta idea se puede encontrar en Black (1910), p. 955: "The word is also commonly used to denote any external object over which the right of property is exercised".

9 En esta dirección, véase, por ejemplo, PENNER (1997). 
se puede argumentar que, en realidad, la propiedad no es un solo derecho, sino una pluralidad de derechos. Básicamente, lo que puede leerse inicialmente como una aclaración de la noción de uso exclusivo de un bien puede considerarse indicativo del hecho de que identificar la propiedad con un solo derecho no es suficiente para dar cuenta satisfactoriamente de ella. En cambio, es necesario aceptar que la propiedad es una acumulación de múltiples derechos (por ejemplo, el derecho a consumir el bien, el derecho a destruirlo y el derecho a la exclusión de otros del acceso al bien), diferentes entre sí por sus contenidos. Si la propiedad se puede describir como una pluralidad de derechos, la afirmación de que la propiedad es un único derecho puede considerarse como una afirmación hecha por razones de simplificación. Por lo tanto, podría decirse que solo a primera vista la propiedad es un solo derecho y que, en realidad, es una pluralidad de derechos, reunidos y simultáneamente ostentados por la misma persona. ${ }^{10}$ Por lo tanto, el bien designado por el término "propiedad" en su segundo uso sería el objeto de esta pluralidad de derechos.

Si creemos que la propiedad es un solo derecho o que la propiedad es una pluralidad de derechos, reconoceremos que el contenido de este derecho o de estos derechos determina una regulación de las relaciones entre el titular del derecho o de los derechos y otras personas en conexión con uno o más bienes. Por consiguiente, tanto si uno identifica la propiedad con un solo derecho o con múltiples derechos, la función de ese derecho o esos derechos siempre será descriptible como la regulación las relaciones entre personas en conexión con uno o más bienes.

Por lo tanto, las declinaciones de la tesis de que la propiedad puede ser descrita mediante la noción de derecho son dos:

1. la propiedad es un solo derecho que regula las relaciones entre personas en conexión con uno o más bienes;

2. la propiedad es una pluralidad de derechos (que, solo por razones de simplificación, pueden describirse como un único derecho) que regulan las relaciones entre personas en conexión con uno o más bienes.

Es posible criticar la segunda declinación de esta tesis, señalando que la posición del titular de los derechos no es equivalente a un grupo de posiciones jurídicas subjetivas que solo son activas (como los derechos), sino también

10 Una tesis de este tipo se puede encontrar en HoHFELD (1917), p. 746, en relación con el caso de un fee-simple owner: "Suppose, for example that $A$ is fee-simple owner of Blackacre. His 'legal interest' or 'property' relating to the tangible object that we call land consists of a complex aggregate of rights (or claims), privileges, powers, and immunities". 
pasivas, como, por ejemplo, el deber de no usar el bien de una manera que sea perjudicial para otras personas o la sujeción a la ejecución o a la tributación. Sobre la base de estas observaciones, es posible avanzar la idea de que la propiedad en realidad equivaldría a una pluralidad de posiciones jurídicas subjetivas, que no son solo derechos (es decir, posiciones jurídicas subjetivas activas), sino también posiciones jurídicas subjetivas pasivas. ${ }^{11}$ La función de estas posiciones es, todavía, descriptible como la regulación de las relaciones entre personas en conexión con uno o más bienes. Una consecuencia de esta idea es que, cuando usamos el término "propiedad" para designar un bien, estamos designando el bien sobre el cual existen todas estas posiciones jurídicas.

La tesis que se acaba de describir, así como las otras dos tesis mostradas anteriormente, es una declinación de la tesis de que la propiedad puede ser descrita mediante la noción de posición jurídica subjetiva, ya sea que esta posición sea un derecho o no.

\section{LA PROPIEDAD COMO UNA O MÁS NORMAS O REGLAS}

Hasta ahora he examinado la tesis de que la propiedad puede explicarse a través de una apelación (que toma diferentes formas) a la noción de posición jurídica subjetiva. Una posición alternativa es que la propiedad coincide con una o más normas o reglas, que, como las posiciones jurídicas subjetivas empleadas por esa tesis, pueden ser vistas como dotadas de la función de regular las relaciones entre personas en relación con uno o más bienes. ${ }^{12}$ Esta tesis explica la propiedad no a través de la noción de posición jurídica subjetiva, sino a través de la noción de norma o regla. Sobre la base de esta tesis, el término "propiedad", cuando se utiliza en la segunda de las formas descritas anteriormente, designa el bien en conexión con el cual las relaciones entre personas son reguladas por una o más normas o reglas.

Hay que añadir algo sobre la relación entre la tesis de la propiedad como una o más posiciones jurídicas subjetivas y la tesis de la propiedad como una o más normas o reglas. La norma o regla o las normas o reglas que se acaba de

11 Para una caracterización de este tipo, considérese el famoso análisis propuesto por HonOrÉ (1961), en que se apela a una lista de posiciones jurídicas subjetivas (vistas como standard incidents).

12 Para una tesis de este tipo, véanse SNARE (1972) y WALdRon (1985), p. 318: “[t] he concept of property is the concept of a system of rules governing access to and control of material resources". 
mencionar pueden considerarse como la fuente del derecho que, según la primera declinación de la primera tesis, coincide con la propiedad, o como la fuente de las posiciones jurídicas subjetivas que, según la segunda declinación de la misma tesis, coinciden con la propiedad. La diferencia entre las dos tesis es, por lo tanto, una cuestión de perspectiva, ya que depende de la elección de privilegiar la noción de posición jurídica subjetiva o la noción de norma o regla en la explicación de la propiedad. En el primer caso, daremos valor a lo que da lugar a una o más de las posiciones jurídicas subjetivas existentes en un ordenamiento jurídico (esto es, a una o más de las normas o reglas que lo componen), en el segundo caso, en cambio, a lo que se originó a partir de una o más de las normas o reglas de un ordenamiento jurídico (esto es, a una o más de las posiciones jurídicas subjetivas ostentadas por los miembros de ese ordenamiento).

Por lo tanto, se podría decir que estas dos tesis son dos descripciones diferentes del mismo fenómeno. ¿Hay, podría preguntarse en este punto, otra descripción que pueda describir ambos? Si la respuesta a esta pregunta es afirmativa, la descripción en cuestión debe constituir una descripción de propiedad compartida por las dos tesis.

\section{UNA BASE COMÚN}

Ahora es necesario comprender si las diferentes tesis sobre la propiedad descritas anteriormente comparten algo entre sí. ¿Hay elementos en común entre las dos descripciones generales de la propiedad que he considerado?

Como se ha dicho, desde el primer punto de vista, la propiedad es un grupo de posiciones jurídicas subjetivas o una sola posición jurídica subjetiva (más específicamente, un derecho) con la función de regular las relaciones entre personas en conexión con uno o más bienes. En cambio, desde el segundo punto de vista, la propiedad coincide con una o más normas o reglas que regulan las relaciones entre personas en conexión con uno o más bienes. En ambos casos, por lo tanto, la función de las entidades identificadas con la propiedad es la misma: regular las relaciones entre personas en conexión con uno o más bienes; y esto es el primer elemento común a ambas descripciones (tanto a ella de la primera tesis como a aquella de la segunda tesis). Por eso, la diferencia entre los efectos de las dos tesis está solo en la diversidad de las cosas identificadas con la propiedad; la función de estas cosas es la misma.

Ahora centrémonos en el tipo de cosas que realizan esta función en ambos casos. En el primer caso, se trata de posiciones jurídicas subjetivas, mientras, en el segundo caso, son normas o reglas. ¿Hay algún elemento que sea común 
a estos dos tipos de entidades? La respuesta es afirmativa. Cuando tratamos de describir una norma o regla, así como cuando tratamos de describir una posición jurídica subjetiva, necesariamente debemos recurrir a expresiones como "obligatorio", "permitido", "prohibido" y similares. Las nociones que debemos utilizar, a fin de hablar de normas o reglas y de posiciones jurídicas subjetivas, son, por lo tanto, al menos, modalidades deónticas (o sea, al menos, obligatorio, permitido y prohibido). Estas modalidades son los "bloques de construcción" tanto de la noción de posición jurídica subjetiva como de la noción de norma o regla. Obviamente, esto no es lo mismo que decir que la noción de norma o regla y la noción de posición jurídica subjetiva son equivalentes: simplemente, ambas nociones se pueden describir mediante el uso de modalidades deónticas. ${ }^{13}$ Una pregunta que podría surgir en este punto es: ¿cuántas modalidades deónticas?

La respuesta es que, dependiendo de las características de la tesis relativa a la propiedad que se elija adoptar, se necesitará una modalidad deóntica o más de una modalidad deóntica. $\mathrm{Si}$, por ejemplo, se declarara que la propiedad es el derecho al uso exclusivo de un bien, podríamos describir este derecho utilizando una sola modalidad deóntica, es decir, diciendo que está permitido solo a un individuo usar ese bien. En otros casos, se podría utilizar una pluralidad de modalidades deónticas, por ejemplo, cuando queremos describir un grupo de diferentes posiciones jurídicas subjetivas o un grupo de diferentes normas o reglas que no son todas descriptibles solo mediante el uso de "permitido". Entonces, ambos casos se pueden describir hablando del uso de un conjunto de una o más modalidades deónticas.

Por lo tanto, si combinamos los dos puntos compartidos que he descrito, podemos ir tan lejos como para decir que la propiedad siempre se puede describir como un conjunto de una o más modalidades deónticas que regulan las relaciones entre personas en conexión con uno o más bienes: esta es la idea que une las dos concepciones de propiedad que he examinado. Entonces, el sentido mínimo de "propiedad" se puede considerar como un conjunto de una o más modalidades deónticas que regulan las relaciones entre personas en conexión con uno o más bienes.

13 Un tratamiento de la propiedad que destaca la conexión entre las modalidades normativas y las posiciones jurídicas subjetivas, así como la conexión entre ellas y la imagen de la propiedad como un "bundle of sticks", se puede encontrar en Munzer (1990), p. 23: "The idea of property [...] involves a constellation of Hohfeldian elements, correlatives, and opposites; a specification of standard incidents of ownership and other related but less powerful interests; and a catalog of 'things' (tangible and intangible) that are the subjects of these incidents. Hohfeld's conceptions are normative modalities. In the more specific form of Honoré's incidents, these are the relations that constitute property. Metaphorically, they are the 'sticks' in the bundle called property". 
Es importante tener en cuenta que identificar la propiedad con un conjunto de una o más modalidades deónticas que regulan las relaciones entre una persona y un bien no es más que una forma más sintética de expresar la idea de que la propiedad es un conjunto de una o más modalidades deónticas que regulan las relaciones entre esa persona y otras personas en conexión con ese bien. La razón de esto es que la relación regulada por una o más modalidades deónticas entre una persona y un bien no es otra cosa que la relación regulada por una o más modalidades deónticas entre esa persona y otras personas en conexión con ese bien.

Obviamente, es posible confiar en este sentido mínimo para explicar los dos usos del término "propiedad" vistos al principio. En primer lugar, ese algo designado por el término "propiedad" en su primer uso corresponde a un conjunto de una o más modalidades deónticas que regulan las relaciones entre personas en conexión con uno o más bienes. El segundo uso del término, en cambio, puede explicarse como un modo de designar el bien en conexión con el cual las relaciones entre personas están reguladas por un conjunto de una o más modalidades deónticas.

\section{CONCEPTO Y CONCEPCIONES DE PROPIEDAD}

Es posible expresar las conclusiones recién alcanzadas utilizando algunas nociones más familiares, a saber, las nociones de concepto y concepción, ya empleadas en el estudio de la noción de propiedad en la filosofía del derecho. ${ }^{14}$ Se puede afirmar que el sentido mínimo del término "propiedad" es el concepto de propiedad, mientras que las descripciones de propiedad ofrecidas por las dos tesis descritas anteriormente son concepciones de propiedad. Comparten el concepto de propiedad, pero lo configuran de diferentes maneras, porque califican la modalidad deóntica o las modalidades deónticas del conjunto que es el concepto de propiedad (i.e., modalidades deónticas que regulan las relaciones entre personas en conexión con uno o más bienes) como elementos constitutivos de diferentes entidades: ya sea como los bloques de construcción de una o más posiciones jurídicas subjetivas o como los bloques de construcción de una o más normas o reglas. Esto significa que la razón por la cual las descripciones de la propiedad propuestas por las dos tesis descritas anteriormente pueden ser calificadas como concepciones de propiedad es solo que el sentido mínimo del término "propiedad" aquí propuesto es más básico y genérico que los sentidos asociados al término "propiedad" por esas dos tesis.

14 Véase WALDRON (1985). Hay otros importantes usos de la distinción entre concepto y concepción: véanse, por ejemplo, RaWLs (1971), pp. 5-6, y DWORKIN (1977), pp. 134-136. 
La diferencia entre concepto y concepciones de propiedad se puede emplear también para otro propósito: no solo se puede usar, como se acaba de hacer, para calificar las diferentes conclusiones producidas por teorías sobre el significado del término "propiedad" como diferentes concepciones de propiedad, sino también para calificar como tales las diferentes formas en que la propiedad ocurre en diferentes ordenamientos jurídicos. Los diferentes ordenamientos jurídicos presentan formas de propiedad más o menos diferentes, pero todos ellos se pueden describir utilizando el concepto de propiedad. Pueden diferir en el modo en que las modalidades deónticas contenidas en el conjunto identificable con el concepto de propiedad se utilizan para regular las relaciones entre personas en conexión con uno o más bienes: elegir, para regular las relaciones entre personas en conexión con uno o más bienes, cuántas y qué modalidades deónticas usar y a qué (esto es, a qué conducta humana) deben aplicarse significa crear una forma específica de propiedad, que se puede calificar como una concepción de propiedad.

Un problema que se puede abordar en este punto es que los bloques de construcción del concepto de propiedad se pueden considerar como los bloques de construcción de otros conceptos. Por ejemplo, el concepto de usufructo y el concepto de hipoteca se pueden describir como conjuntos de una o más modalidades deónticas que regulan las relaciones entre personas en conexión con uno o más bienes. Esto parece hacer imposible distinguir cosas como usufructo e hipoteca de la propiedad (pero también distinguir cosas como usufructo e hipoteca entre sí) y, sin embargo, parece absurdo negar la existencia de la posibilidad de una distinción en este caso. El punto que se debe destacar es que la distinción en cuestión tiene lugar en un nivel diferente del nivel en el que se colocan los conceptos, es decir, los sentidos mínimos de términos como "propiedad". Es posible distinguir la propiedad del usufructo tan pronto como se abandona el nivel de los conceptos y se mueve a un nivel en el que conceptos se declinan de diferentes maneras.

Todo esto hace evidente que la descripción de propiedad aquí propuesta no debe considerarse como una definición que proporciona las condiciones necesarias y suficientes para cada uso del término "propiedad".

\section{LO QUE ES EXTERNO A LA PROPIEDAD}

Ahora es el momento de considerar un problema específico relacionado con el tratamiento de la propiedad aquí propuesto, es decir, el problema de lo que es externo a la propiedad: dado el concepto de propiedad descrito anteriormente, ¿cuáles son los elementos que, aunque relevantes para el contenido de 
este concepto, pueden considerarse externos a él? Tales elementos se pueden encontrar al centrarse en dos cuestiones diferentes.

La primera cuestión se refiere a la relación entre el concepto de propiedad y las nociones de persona y de bien. Es importante subrayar que personas y bienes son elementos constitutivos del concepto de propiedad, pero este concepto no ofrece una definición de "persona" y de "bien". Esto significa que los criterios para identificar personas y bienes son externos al concepto de propiedad. Este concepto es un conjunto de una o más modalidades deónticas que regulan las relaciones entre personas en conexión con uno o más bienes, pero no nos dice qué entidades están calificadas como personas y qué entidades están calificadas como bienes. No es la propiedad lo que hace de algo una persona o un bien; más bien, una vez que se ha establecido qué entidades son personas y qué entidades son bienes, es posible aplicar el concepto de propiedad, es decir, emplear un conjunto de una o más modalidades deónticas para regular las relaciones entre esas entidades que han sido calificadas como personas en conexión con esas entidades que han sido calificadas como bienes.

El segundo tema que quiero discutir es similar al tema considerado por August Thon, que se preguntó si el poder que transfiere la propiedad se podría considerar como una parte de la propiedad misma. ${ }^{15}$ La respuesta de Thon fue negativa. Para apoyarla, empleó el siguiente ejemplo: si arrojo una piedra de un punto a otro, lo que transfirió la piedra es una fuerza, pero nadie diría que esta fuerza vino de la piedra; en consecuencia, esta fuerza debe ser algo externo a la piedra. Este ejemplo mostraría que lo que transfiere (en el ejemplo, la fuerza) no puede ser, al mismo tiempo, lo que se transfiere (en el ejemplo, la piedra). Debido a esto, se puede decir que el poder que transfiere la propiedad no puede ser parte de la propiedad, así como el poder que transfiere una piedra no puede ser parte de esa piedra.

Aquí no quiero examinar críticamente los argumentos formulados por Thon. Me limito a señalar que un problema similar al enfrentado por Thon surge de una de las tesis que se han ilustrado previamente. Más precisamente, el problema en cuestión surge si seguimos una de las formas de la tesis de la propiedad como una o más posiciones jurídicas subjetivas y consideramos la propiedad como un grupo de derechos. Dados estos supuestos, podemos preguntarnos si el derecho a transferir propiedades está incluido en ese grupo.

15 Thon (1878), pp. 327-328. 
La respuesta afirmativa puede ser problemática: si suponemos, por ejemplo, que la propiedad es un grupo que incluye los derechos $\mathrm{X}, \mathrm{Y}$ y $\mathrm{Z}$, y que $\mathrm{Z}$ es el derecho a transferir la propiedad, $Z$ tiene como objeto la propiedad, i.e., los derechos $\mathrm{X}, \mathrm{Y}$ y Z, lo que significa que este caso es un caso de autorreferencia parcial, debido al hecho de que $\mathrm{Z}$ también se refiere a sí mismo. Una manera de evitar la autorreferencia parcial de este caso es afirmar que una caracterización de la propiedad como la que se acaba de presentar no es del todo correcta: el error estaría en pensar que $Z$ es un miembro del grupo que coincide con la propiedad. Si se afirma que $Z$ es un derecho que no es un elemento constitutivo de la propiedad, se elimina el problema de la autorreferencia parcial, porque $\mathrm{Z}$ se convierte en un derecho externo a la propiedad.

\section{CONCLUSIONES}

En este punto, es posible repetir brevemente las tesis respaldadas hasta aquí y formular algunas conclusiones generales que se pueden sacar de ellas.

He comenzado con la distinción entre tres problemas relativos a la propiedad. Al concentrarme en uno de ellos (el problema de qué es la propiedad), he distinguido dos tesis que constituyen dos modos diferentes de resolverlo: la tesis de la propiedad como una o más posiciones jurídicas subjetivas y la tesis de la propiedad como una o más normas o reglas. He argumentado que estas tesis pueden verse como descripciones diferentes del mismo fenómeno y que hay una descripción más general de ello, que es el sentido mínimo del término "propiedad" (un conjunto de una o más modalidades deónticas que regulan las relaciones entre personas en conexión con uno o más bienes). Basándome en esta descripción, he intentado explicar el segundo uso del término "propiedad" (a través del cual se designa un bien). Finalmente, he usado las nociones de concepto y concepción y las he aplicado a la propiedad, sosteniendo que el sentido mínimo del término "propiedad" que he descrito es el concepto de propiedad, mientras que las concepciones de propiedad son las descripciones de la propiedad proporcionadas por las dos tesis descritas, pero también las formas de propiedad creadas en los diferentes ordenamientos jurídicos.

En este punto, uno podría preguntarse qué utilidad tiene encontrar el concepto de propiedad. Quizás sería más útil indagar sobre formas individuales de propiedad o examinar con mayor profundidad los criterios en virtud de los cuales ciertas cosas se califican como bienes o personas, o los criterios en virtud de los cuales se aplica el concepto de propiedad. Ciertamente, el estudio de estos temas es fundamental, pero encontrar el concepto de propiedad puede no considerarse inútil, ya que nos ayuda a entender por qué sentimos que, aunque el término 
"propiedad" se puede usar en diferentes modos en el lenguaje jurídico y en el lenguaje ordinario, hay algo que es común a sus diferentes usos considerados aquí. Encontrar el concepto de propiedad hace posible comprender qué es este elemento común. Esto, a su vez, permite comprender dentro de qué límites semánticos se mueven los debates sobre el significado del término "propiedad". Una definición del término "propiedad" que no puede describirse en los términos del concepto de propiedad representaría un intento de innovación, un escape de los límites en cuestión. 


\section{BIBLIOGRAFÍA CITADA}

Becker, Lawrence C. (1977): Property Rights. Philosophic Foundations (Routledge \& Kegan Paul).

Bentham, Jeremias (1821): Tratados de legislación civil y penal, Tomo I (trad. Ramón Salas, Imprenta de D. Fermín Villalpando).

Black, Henry Campbell (1910): A Law Dictionary (West Publishing Co., 2 ${ }^{\mathrm{a}}$ ed.).

Cohen, Félix S. (1954): "Dialogue on Private Property", Rutgers Law Review, Vol. 9, No 2, pp. 357-387.

Dworkin, Ronald (1977): Taking Rights Seriously (Harvard University Press).

Hart, H. L. A. (1968): Punishment and Responsibility. Essays in the Philosophy of Law (Clarendon Press).

HoHFELD, Wesley Newcomb (1917): "Fundamental Legal Conceptions as Applied in Judicial Reasoning", The Yale Law Journal, Vol. 26, № 8, pp. 710-770.

Honoré, A. M. (1961): “Ownership”, en Guest, A. G. (ed.), Oxford Essays in Jurisprudence (Oxford University Press), pp. 107-147.

JENKs, Edward (1932): "Bentham's Theory of Legislation", Journal of Comparative Legislation and International Law, Vol. 14, No 4, pp. 289-291.

MunzeR, Stephen R. (1990): A Theory of Property (Cambridge University Press).

Penner, J. E. (1997): The Idea of Property in Law (Clarendon Press).

Rawls, John (1971): A Theory of Justice (Harvard University Press).

SnARE, Frank (1972): “The Concept of Property", American Philosophical Quarterly, Vol. 9, № 2, pp. 200-206. 
Thon, August (1878): Rechtsnorm und subjectives Recht. Untersuchungen zur allgemeinen Rechtslehre (Hermann Böhlau).

WALDRON, Jeremy (1985): “What Is Private Property?”, Oxford Journal of Legal Studies, Vol. 5, № 3, pp. 313-349. 


\title{
RESEÑA: RELIANCE IN THE BREAKING-OFF OF CONTRACTUAL NEGOTIATIONS
}

\author{
IÑIGO DE LA MAZA GAZMURI*
}

Isabel ZuLOAGA, Reliance in the Breaking-Off of

Contractual Negotiations. Trust and Expectation in a

Comparative Perspective (Intersentia, 2019).

\section{EN LOS EXTREMOS MORA EL EXCESO ${ }^{1}$}

En Walford $v$ Miles, ${ }^{2}$ una sentencia de la House of Lords de 1992, Lord Ackner señaló lo siguiente:

(...) la idea de un deber de llevar a cabo negociaciones de buena fe es inherentemente contraria a la posición adversarial que adoptan las partes que se encuentran negociando. Cada parte de las negociaciones tiene derecho a perseguir su propio interés, siempre y cuando no proporcionen representaciones falsas. ${ }^{3}$

Por su parte, a propósito de los deberes precontractuales de información, el profesor italiano Francesco Galgano ha indicado que:

En estos casos la buena fe asume, sobre todo, el carácter de un deber de información de una parte respecto de la otra: cada una de ellas tiene el deber de informar las circunstancias que sean desconocidas para la otra y que puedan resultar determinantes para la prestación de su consentimiento (aquellas por las que la otra parte, si las hubiese sabido, no habría contratado o habría contratado bajo condiciones diversas). Así, por ejemplo, es contrario a la buena fe no decir mientras se está vendiendo un terreno edificable que se tiene conocimiento de que un proyecto de modificación del plan general de ordenación urbana modificará el destino de esa área...; o bien (en la hipótesis inversa) no decir, mientras se negocia el precio de adquisición de un terreno agrícola, que se tiene conocimiento de la existencia de una modificación del plan general de ordenación urbana, en trámite

\footnotetext{
* Universidad Diego Portales, Chile (inigo.delamaza@udp.cl).

1 Sobre esto ver de la Maza Gazmuri (2020), passim.

2 [1992] 2 AC 128.

3 Walford v Miles (1992), p. 173.
} 
de aprobación, que hace edificable tal terreno lo cual elevaría sensiblemente el precio. ${ }^{4}$

Ambos son extremos, ambos, por lo tanto, deben ser evitados. No es buena política pública tratar a quienes negocian un contrato como si fueran sociópatas, pero tampoco lo es exigirles una conducta propia de un filántropo, in medio veritas est.

Una lectura cuidadosa del libro en que la profesora Isabel Zuloaga volcó su tesis doctoral suministra buenos insumos para pensar las cosas de esta manera, resultando de utilidad para conjurar los riesgos que entrañan tanto los arrebatos de la buena fe como el escepticismo del derecho inglés frente a ella.

\section{LA ZONA DE PENUMBRA}

Según anuncia Zuloaga al comienzo de su libro, sus esfuerzos analíticos se limitan a una de las posibles variaciones del retiro unilateral de las tratativas preliminares; se trata de lo que denomina el "caso paradigmático" (paradigm case) (p. xxxiv). Este caso tiene lugar toda vez que existiendo una negociación (que no ha culminada en ningún tipo de contrato, ni preparatorio ni definitivo) en la cual han existido gastos y sin que exista intención de dañar, una de las partes, que ha suscitado en la otra la otra parte la confianza de que el contrato se perfeccionará, se retira (p. xxxiv).

En mi opinión lo que hace realmente interesante en términos de responsabilidad civil un escenario como el que ofrece la autora es que se encuentra -para utilizar con cierta libertad un término hartiano- en una "zona de penumbra" entre la responsabilidad contractual y la responsabilidad extracontractual.

4 Galgano (1992), p. 461 (con todo, una mirada sobre la opinión del mismo autor en la misma obra sobre el dolo por omisión muestra una opinión más ponderada, en p. 309). En el mismo sentido, a propósito de la cognoscibilidad del error, Pietrobon ha sostenido que: "La aplicación general del principio de la buena fe debería conducir a no tutelar la posición, evidentemente desleal, de quien se da cuenta del error ajeno y de la fuerza determinante que aquel tiene sobre la parte contraria y, a pesar de saberlo, calla y concluye igualmente el contrato (Pietrobon, p. 126). En el mismo sentido Betti ha señalado respecto de las tratativas preliminares que "el deber recíproco de lealtad y de probidad prenegocial, que impone eventualmente, no solo deberes negativos, sino también deberes positivos, consistentes en revelar la realidad de las cosas, tal y como cada uno las conoce, según su ciencia y conciencia (BeTTI, p. 92). 
Es cierto, no existe aún un contrato y, por lo mismo, no parece posible exigir a las partes los intensos deberes de colaboración que, bajo la forma de las obligaciones, impone el derecho de contratos. Sin embargo, tampoco se trata de una situación paradigmática de ilícitos extracontractuales, en las cuales, previo a la comisión del ilícito (imaginemos un atropello) no hay relación alguna entre las partes.

De alguna manera, el escenario en que se plantea el caso que interesa a Zuloaga se encuentra en alguna parte del continuo que es posible trazar en los extremos configurados por el escenario típico del contrato (por ejemplo, una compraventa) y del ilícito extracontractual (por ejemplo, un atropello). Y esta ubicación en la penumbra determina que, ni el enfoque propio de la colaboración entre las partes que engendra el perfeccionamiento de un contrato ni aquel de indiferencia que suele caracterizar a la responsabilidad extracontractual sean completamente adecuados aquí.

Y, entonces, si no me equivoco, lo que Zuloaga pretende es alumbrar esa penumbra a través de la idea de confianza.

\section{EL DISCRETO ENCANTO DEL MÉTODO COMPARATIVO}

Se trata de una empresa extremadamente atractiva y el resultado es uno por el cual se debe felicitar a la autora, entre otras cosas, porque no ofrece más de lo que está dispuesta a dar.

El enfoque que emplea Zuloaga es el propio de una de las versiones del método comparativo que le permite mostrar que, respecto de su caso paradigmático, no existe equivalencia funcional entre la aproximación de los derechos alemán, francés y chileno por una parte y el inglés por la otra.

Desde luego existe un enorme valor en lo que la autora logra. Una cosa es afirmar algo así -que parece relativamente intuitivo- otra es acreditarlo con el esmero y claridad que logra la autora.

Sin embargo -y en esto no hay crítica, sino, simplemente, una constatación- existen otros extremos que quedan más abandonados. Así, por ejemplo, la caracterización de las negociaciones desde una perspectiva más multidisciplinaria (económica, filosófica, sociológica, etc.) que contribuya a evaluar la adecuación de la idea de confianza como un dispositivo que ilumine la zona de penumbra. 
Por otra parte, la idea de confianza, que viene a desarrollar un enorme trabajo en la tesis de Zuloaga es, según me parece, extremadamente correcta y la distinción entre "trust dimension" y "expectation dimension" sugerente, aunque, para efectos de su trabajo la única relevante sea la segunda. Igualmente, aunque no sea una idea original, es correcto que el reproche consiste en crear una apariencia que luego se defrauda. Igualmente, existen páginas valiosas dedicadas a la legitimidad de la confianza pues, aunque escasamente teóricas, indican circunstancias objetivas a partir de las cuales puede extraerse dicha legitimidad (pp. 156-158). Se echa en falta, sin embargo, una articulación entre la legitimidad de la confianza y la falta de justificación del retiro pues, como sucede en todos los ordenamientos jurídicos, la responsabilidad exige imputabilidad subjetiva. Es cierto, dicha imputabilidad subjetiva puede procurarse a través de la creación de confianza, pero sucede que lo que gatilla la responsabilidad es el retiro, por lo mismo, parece necesario un estudio acerca del carácter justificado o injustificado del retiro que yo no he sabido encontrar en la tesis. ${ }^{5}$

En fin, es muy probable que aún se pueda añadir algo sobre el tratamiento de la buena fe; señaladamente que la distinción entre buena fe objetiva y subjetiva parece ser completamente digna de abandono y, en su lugar, establecida una distinción entre el principio general y sus manifestaciones. También siembra dudas en un lector continental la idea de que pueda abandonarse el uso de la buena fe en el periodo de las tratativas preliminares y reemplazarlo por la confianza (p. 165). En realidad, según me parece, un lector continental señalaría que la confianza es la forma que asumen las exigencias que formula el principio general de la buena fe frente a la cuestión de las tratativas preliminares. Muy probablemente, a ese lector el abandono de la buena fe le parecería más bien fútil, salvo que se le explicara que, de esa manera, es más probable que sea aceptada por el derecho inglés.

En fin, sirviéndose del método comparativo, la profesora Isabel Zuloaga ha realizado una obra valiosa tanto para lectores del mundo continental como respecto de aquellos del common law. Para volver al principio, al presentar las exigencias de la buena fe durante el periodo contractual de una forma mesura$\mathrm{da}$, que equilibra las posiciones, eventualmente adversariales, de ambas partes, conjura el riesgo de la posición filantrópica ilustrada por Galgano. De otra parte, al lector del common law le enseña que, quizás, la inherente repugnancia que sentía Lord Ackner por la buena fe se atenúe si se presenta de una manera sobria e inteligente como lo hace la profesora Zuloaga.

5 Se trata, pero de manera extremadamente leve, en las páginas 148-149. 


\section{BIBLIOGRAFÍA CITADA}

BetTI, Emilio (1969). Teoría general de las obligaciones (Editorial Revista de Derecho Privado).

De la Maza Gazmuri, Iñigo (2020). "El retiro unilateral de las negociaciones en los PLDC", en VIDAL, Alvaro y SEVERIN, Gonzalo (eds.), La armonización del derecho de contratos en Latinoamérica (Thomson Reuters), pp. 123-140.

Galgano, Francesco (1992), El negocio jurídico (Tirant lo Blanch).

Pietrobon, Vittorino (1971). El error en la doctrina del negocio jurídico (trad. M. Alonso Pérez, Editorial Revista de Derecho Privado).

Zuloaga, Isabel (2019). Reliance in the Breaking-Off of Contractual Negotiations. Trust and Expectation in a Comparative Perspective (Intersentia). 